Dennoch gibt das Buch in Bezug auf die im Titel angekündigten „Prinzipien globaler Sicherheit" wenig her. Überzeichnend gesagt erscheint es über weite Strecken so, als sei Steinbruners Vorstellung von diesen Prinzipien die folgende: Die USA sind und bleiben der Nabel der Welt, und die Herausforderungen der Globalisierung stellen keine kritischen Fragen an das traditionelle Selbstbild der Amerikaner, sondern rufen nur nach neuen Methoden, um diesem Selbstbild gerecht bleiben zu können. Eine Zusammenstellung von und kritische Auseinandersetzung mit politischen Kriterien für globale Sicherheit und militärische Aktionen zur Verwirklichung globaler Sicherheit sucht man in Steinbruners Argumentation weitestgehend vergebens. Der Leser muss sich mit lapidaren Feststellungen zufrieden geben, wie etwa, dass menschliche Gesellschaften nicht unbegrenzt dem Prinzip der Nichtintervention in die inneren Angelegenheiten eines Staates folgen können, wenn innere Unruhen die Funktionsregeln der Weltwirtschaft bedrohen (139) oder wenn das innerstaatliche Rechtssystem „versagt" hat (149).

Gleichwohl ist die Quintessenz von Steinbruners Untersuchung in erster Linie ein politisches Plädoyer: Die USA können ihre nationale Sicherheit nicht länger durch Unilateralismus und Dominanz garantieren, sondern es ist - unabhängig davon, welche politische Strömung gerade vorherrscht - entscheidend, dass sie sich auf internationale Kooperation und Multilateralismus einlassen. Und zwar nicht nur rhetorisch, sondern auch in der Verteidigungsplanung und bei der Reform der Streitkräfte. Auf alle Fälle ist Steinbruners Beitrag ein Beispiel dafür, wie man sich mit den Herausforderungen der Globalisierung (hier im Bereich Sicherheitspolitik) politikwissenschaftlich sehr ernst zu nehmend und analytisch tief greifend auseinander setzen kann, ohne die Praxisrelevanz zu vernachlässigen und ,Kundeninteressen' zu ignorieren. Davon kann gerade die deutsche Globalisierungs-Forschung viel lernen.
Ernst-Otto Czempiel: Kluge Macht: Außenpolitik für das 21. Jahrhundert. München: C.H. Beck 1999, 274 S., DM 48,-.

Monika Medick-Krakau (Hg.): Außenpolitischer Wandel in theoretischer und vergleichender Perspektive: Die USA und die Bundesrepublik Deutschland. BadenBaden: Nomos Verlagsgesellschaft 1999, 313 S., DM 89,-.

\section{Sebastian Bartsch}

Wenn zu Ehren eines der Gründungsväter der Internationalen Beziehungen in der Bundesrepublik eine Festschrift erscheint und der Geehrte fast zeitgleich ein eigenes Buch vorlegt, dann ist dies eine interessante Koinzidenz. Wenn sich beide Veröffentlichungen zudem mit einem Thema beschäftigen, das bislang sicherlich kein Aushängeschild der Disziplin gewesen ist - die Rede ist von der Außenpolitikanalyse und vom Wandel der Außenpolitik -, dann ist dies ein weiterer Grund, neugierig zu sein.

Monika Medick-Krakau verfolgt mit dem von ihr herausgegebenen Band das Interesse, Folgen des Wandels in der internationalen Umwelt für außenpolitische Grundorientierungen und Strategien zu identifizieren. Mit Blick auf die Bundesrepublik und die USA sollen „Bausteine für einen Vergleich“ erarbeitet werden, der ein besseres Verständnis von Kontinuität und Veränderung in der Außenpolitik beider Länder ermöglicht. Diese Zielsetzung ist wohlweislich vorsichtig formuliert, denn ein ambitionierteres Unterfangen hätte angesichts des von der Herausgeberin eingangs höchst konzentriert beschriebenen, bescheidenen Standes der Forschung leicht Schiffbruch erleiden können. Herausgekommen ist denn auch kein systematischer Vergleich, sondern ein, und dies ist eben die Kehrseite der offenen Struktur des Buches, sehr heterogener Mix von Texten, die sich dem Thema aus unterschiedlichsten Richtungen und mit jeweils spezifischen Schwerpunkten nähern.

Wie ein möglicher theoretischer Rahmen für den Band hätte aussehen können, lässt Thomas Risses Aufsatz über „sozialkonstruktivistische Perspektiven zum Wandel in der Außenpolitik" erkennen. Ohne für den Sozialkonstruktivismus den Anspruch einer substanziellen Theorie außenpolitischen Wandels zu erhe- 